Quim. Nova, Vol. 32, No. 4, 868-874, 2009

\title{
CARACTERIZAÇÃO DE SUBPRODUTO DA INDÚSTRIA DE ALUMÍNIO E SEU USO NA RETENÇÃO DE CÁDMIO E CHUMBO EM SISTEMAS MONOELEMENTARES
}

\author{
Enio Tarso de Souza Costa*, Luiz Roberto Guimarães Guilherme, Nilton Curi, Guilherme Lopes e Elton Luiz Visioli \\ Departamento de Ciência do Solo, Universidade Federal de Lavras, CP 37, 37200-000 Lavras - MG, Brasil \\ Luiz Carlos Alves de Oliveira \\ Departamento de Química, Universidade Federal de Lavras, CP 37, 37200-000 Lavras - MG, Brasil
}

Recebido em 10/3/08; aceito em 23/10/08; publicado na web em 6/3/09

\begin{abstract}
CHARACTERIZATION OF AN ALUMINUM INDUSTRY BY-PRODUCT AND ITS USE ON CADMIUM AND LEAD RETENTION IN MONOELEMENTARY SYSTEMS. The present work aimed to characterize an aluminum industry by-product in natura (L.A. nat) and after phosphate and thermal pretreatments; evaluate the adsorption/desorption capacity of $\mathrm{Cd}$ and $\mathrm{Pb}$ by this L.A. nat form and after the aforementioned pretreatments, comparing them with an in natura iron mining by-product (L.F. nat). The L.A. nat presented a high $\mathrm{pH}$ as well as a high $\mathrm{Na}$ concentration and also an oxide-rich mineralogy. Pretreatment of the by-product had no significant effect upon $\mathrm{Cd}$ and $\mathrm{Pd}$ adsorption/desorption. The L.A. nat performed better than the L.F. nat as an $\mathrm{Cd}$ and $\mathrm{Pb}$ adsorbent.
\end{abstract}

Keywords: pretreatment; amendment; trace elements.

\section{INTRODUÇÃO}

A lama da mineração de alumínio (red mud) é um resíduo gerado em grandes proporções pela extração do alumínio da bauxita via processo Bayer. Não existem relatos na literatura de sua quantidade gerada no Brasil, porém consta no Anuário Mineral Brasileiro de 2005 publicado pelo Departamento Nacional de Produção Mineral (DNPM) no ano de 2006 que a produção bruta do minério com um teor de $35,30 \%$ de $\mathrm{Al}_{2} \mathrm{O}_{3}$ foi de 31,19 milhões de $t$, resultando em cerca de 11,01 milhões de t do metal. ${ }^{1}$ Considerando que $1 \mathrm{t}$ de alumínio extraído resulta na geração de 1,5 t de lama, ${ }^{2}$ estima-se que a produção de lama no Brasil no ano de 2005 foi de aproximadamente 16,52 milhões de $t$.

Este material tem sido depositado atualmente em barragens de rejeito, mas existem diversos trabalhos que comprovam sua eficiência como adsorvente de elementos-traço em soluções aquosas ${ }^{3,4}$ e como amenizante de solos contaminados com vários elementos. ${ }^{5-10}$ Seu alto $\mathrm{pH}$ irá proporcionar aumento no $\mathrm{pH}$ dos solos, aumentando a capacidade de adsorção de metais. Uma outra vantagem está na sua constituição muito rica em óxidos, o que lhe imprime a característica de um bom adsorvente. Alguns autores ${ }^{11}$ sugerem que alguns subprodutos industriais ricos em ferro podem ser usados para a remediação de solos contaminados com elementos-traço. A grande maioria desses amenizantes é de natureza alcalina e aumenta o $\mathrm{pH}$ do solo, diminuindo a disponibilidade de $\mathrm{Cd}, \mathrm{Zn}$ e $\mathrm{Cu} .{ }^{12}$ Vale ressaltar que para a utilização de resíduos é indispensável que se faça uma criteriosa caracterização para evidenciar potencialidades e riscos na sua utilização, bem como inferir sobre os possíveis mecanismos que estarão envolvidos nas reações de adsorção e dessorção.

Um resíduo pode ser utilizado como amenizante na sua forma in natura, ou seja, sem passar por nenhum processamento antes do seu uso, o que pode viabilizar as operações por não ter custos adicionais. Porém, com o simples fato de se fazer um pré-tratamento (e.g., aquecimento, adição de ligantes orgânicos ou inorgânicos) pode-se alterar a superfície do material, melhorando sua capacidade como adsorvente. Dessa forma, o pré-tratamento, mesmo tendo um custo adicional, pode se tornar

*e-mail: eniotarso@yahoo.com.br viável por proporcionar melhorias substanciais ao produto bruto.

As superfícies dos constituintes do solo podem ser alteradas com o decorrer do tempo, em função de vários fatores pedogenéticos. Como exemplo, cita-se a transformação da goethita em hematita, via solução, pela dissolução da primeira, liberando ferro, com conseqüente formação da segunda, por precipitação e alteração da estrutura do óxido. ${ }^{13}$ Uma outra forma de modificação dessas superfícies no estado sólido é pela elevação da temperatura, provocando a volatilização de alguns elementos e um rearranjo de toda a estrutura do material.

Pode-se ainda mudar a superfície adsorvente pela alteração do balanço de suas cargas. Por exemplo, ligantes, como o fósforo, na forma de fosfato, quando previamente adsorvidos, podem modificar ou, até mesmo, reverter a carga líquida da superfície de óxidos, de positiva para negativa, e promover a formação de complexos ternários óxido-P-metal. Dessa forma, o fosfato atua como uma ponte entre a superfície do adsorvente e o metal. ${ }^{14}$

Em estudo envolvendo a adsorção e dessorção de cádmio, cobre e chumbo em Latossolos, apresentando balanço de cargas positivas em subsuperfície e submetidos ou não a pré-tratamento com fósforo, verificou-se que este pré-tratamento provocou um aumento na carga líquida negativa (provocado pela redução do ponto de efeito salino nulo), proporcionando um aumento na adsorção de $\mathrm{Cd}, \mathrm{Cu}$ e $\mathrm{Pb}$ e uma redução na quantidade dessorvida de $\mathrm{Pb} .{ }^{15}$ Dada a complexidade do sistema solo, o estudo das reações de sorção em sistemas monoelementares é muito importante para elucidar algumas particularidades que ocorrem com cada elemento nesse sistema.

Considerando-se a importância das reações de adsorção/dessorção em ambientes contaminados, bem como a necessidade de uma boa caracterização de materiais com potencial adsorvente de elementostraço, o presente trabalho teve como objetivos caracterizar um subproduto da indústria de alumínio ( red $m u d$ ), doravante denominado de lama da mineração de alumínio, na sua forma in natura e após ter sido submetida ao pré-tratamento com ácido fosfórico e térmico e, avaliar a capacidade de adsorção/dessorção de cádmio $(\mathrm{Cd})$ e chumbo $(\mathrm{Pb})$ pela lama da mineração de alumínio na sua forma in natura e após ter sido submetida ao pré-tratamento com ácido fosfórico e térmico, comparando-a com a lama da mineração de ferro in natura. 


\section{PARTE EXPERIMENTAL}

Amostras de lama da mineração de alumínio foram coletadas em vários pontos do reservatório da Alcoa em Poços de Caldas e homogeneizadas, obtendo-se uma amostra composta. Esta foi seca ao ar, macerada em gral e passada em peneira de $1 \mathrm{~mm}$ para posteriores pré-tratamentos e análises.

Foram feitos pré-tratamentos com ácido fosfórico e térmico e, tanto estes materiais pré-tratados quanto a lama da mineração de alumínio in natura foram submetidos a análises e testes de adsorção e dessorção com $\mathrm{Cd}$ e $\mathrm{Pb}$ em sistemas monoelementares.

Para o pré-tratamento com ácido fosfórico, pesaram-se $100 \mathrm{~g}$ de lama da mineração de alumínio in natura e adicionaram-se $150 \mathrm{~mL}$ de solução de $\mathrm{H}_{3} \mathrm{PO}_{4}$ (relação lama:solução de 1:1,5), nas seguintes concentrações: $0 ; 21,5 ; 43,0$ e 86,0 mmol de $\mathrm{P} \mathrm{L}^{-1}$. Este pré-tratamento foi realizado tendo como solução de fundo $\mathrm{Ca}\left(\mathrm{NO}_{3}\right)_{2}$ a $5 \mathrm{mmol} \mathrm{L}^{-1}$. As amostras foram agitadas por $72 \mathrm{~h}$, alternando-se $12 \mathrm{~h}$ de repouso e 12 $\mathrm{h}$ de agitação, para que ocorressem as reações de adsorção do fosfato com o adsorvente. Após $72 \mathrm{~h}$, as amostras foram centrifugadas a 3.000 rpm por 30 min e o sobrenadante foi coletado para leitura de fósforo no colorímetro. Após a coleta do sobrenadante, foram adicionados ao material remanescente nos tubos de centrífuga $200 \mathrm{~mL}$ de solução de $\mathrm{Ca}\left(\mathrm{NO}_{3}\right)_{2}$ a $5 \mathrm{mmol} \mathrm{L}^{-1}$ (relação lama:solução 1:2) para que se processassem a lavagem da solução residual da adsorção e a dessorção do fosfato previamente adsorvido. As amostras foram agitadas por mais $12 \mathrm{~h}$, centrifugadas e foi retirado o sobrenadante para leitura de fósforo no colorímetro. Em seguida, o material remanescente nos tubos de centrífuga foi congelado e liofilizado.

Das quantidades adicionadas de fosfato no pré-tratamento da lama da mineração de alumínio $(0,0 \pm 0,0 ; 32,3 \pm 1,6 ; 64,5 \pm 0,2$ e $129,0 \pm 3,5$ mmol $\left.\mathrm{kg}^{-1}\right)$, as quantidades adsorvidas foram de $0,0 \pm 0,0 ; 19,1 \pm 3,9$; $38,5 \pm 0,9$ e $98,7 \pm 8,6 \mathrm{mmol} \mathrm{kg}^{-1} \mathrm{e}$ as quantidades dessorvidas foram de $0,0 \pm 0,0 ; 1,3 \pm 0,0 ; 6,9 \pm 0,3$ e 7,6 $\pm 3,3 \mathrm{mmol} \mathrm{kg}^{-1}$, respectivamente. Descontando-se as quantidades dessorvidas das quantidades adsorvidas, temos que as quantidades de fosfato efetivamente retidas na lama da mineração de alumínio pré-tratada foram de $0,0 \pm 0,0 ; 17,9 \pm 3,9$; $31,6 \pm 1,2$ e $91,1 \pm 11,8 \mathrm{mmol} \mathrm{kg}^{-1}$, respectivamente.

Para o pré-tratamento térmico, pesaram-se $100 \mathrm{~g}$ de lama da mineração de alumínio in natura em cadinhos de porcelana. Estes foram colocados na mufla nas seguintes temperaturas: 105, 300 e 600 ${ }^{\circ} \mathrm{C}$, durante $30 \mathrm{~min}$, contados a partir do equilíbrio da temperatura correspondente a cada tratamento. Em seguida, os cadinhos foram colocados para resfriar em dessecador. Ambos os pré-tratamentos foram feitos em duplicata e, ao final, as amostras foram misturadas, obtendo-se uma amostra composta para posteriores testes de adsorção e dessorção.

Como tentativa de avaliar o uso da lama da mineração de alumínio como substrato, foram preparadas amostras do material na sua forma in natura para análise de fertilidade, segundo método padrão para análise de solo. ${ }^{16}$

Foi feito, nas mesmas amostras, ataque sulfúrico, ${ }^{16,17}$ para a determinação de silício ( $\mathrm{Si}$ ), alumínio (Al), ferro (Fe), titânio (Ti) e fósforo $(\mathrm{P})$, sendo os resultados expressos nas formas de óxidos $\left(\mathrm{SiO}_{2}, \mathrm{Al}_{2} \mathrm{O}_{3}\right.$, $\mathrm{Fe}_{2} \mathrm{O}_{3}, \mathrm{TiO}_{2}$ e $\mathrm{P}_{2} \mathrm{O}_{5}$, respectivamente). A razão molar $\mathrm{Al}_{2} \mathrm{O}_{3} / \mathrm{Fe}_{2} \mathrm{O}_{3}$ foi calculada em função dos valores expressos em percentagens de $\mathrm{Al}_{2} \mathrm{O}_{3}$ e $\mathrm{Fe}_{2} \mathrm{O}_{3}$, divididos pelos seus respectivos pesos moleculares.

As quantidades semitotais dos elementos $\mathrm{Cd}$ e $\mathrm{Pb}$, tanto na lama da mineração de ferro in natura, quanto na lama da mineração de alumínio in natura e após ter sido submetida aos pré-tratamentos com ácido fosfórico e térmico, foram determinadas pela digestão em forno de microondas, segundo o método 3051A da Agência de Proteção Ambiental dos EUA. ${ }^{18}$ Vale ressaltar que as quantidades são ditas semitotais porque o método USEPA 3051A não extrai os elementos presentes nas estruturas de silicatos. As leituras dos extratos foram feitas usando-se espectrofotômetro de absorção atômica de chama (leituras na faixa de concentração em $\mathrm{mg} \mathrm{L}^{-1}$ ) e com forno de grafite (leituras na faixa de concentração em $\mu \mathrm{g} \mathrm{L}^{-1}$ ). O controle de qualidade das análises foi feito por meio do uso de amostras de solo com concentrações de $\mathrm{Cd}$ e $\mathrm{Pb}$ conhecidas, do North American Proficiency Test Program da Soil Science Society of America.

Para a caracterização qualitativa da carga de superfície dos diferentes adsorventes testados, procedeu-se à determinação do ponto de carga zero (PCZ) dos mesmos, por meio da medição da mobilidade eletroforética, usando-se um aparelho Zeta-Meter System 3.0. Foram tomadas as médias de 20 leituras da mobilidade eletroforética nos valores de $\mathrm{pH}$ 1, 3, 5, 7, 9 e 11 para determinar o PCZ.

As amostras dos adsorventes lama da mineração de ferro in natura, lama da mineração de alumínio in natura e após ter sido submetida ao pré-tratamento térmico foram submetidas à difração de raios-X pelo método do pó, ${ }^{19} \mathrm{em}$ aparelho Phillips, modelo PW 1830/40, utilizando a radiação cobalto $\mathrm{K}$ com filtro de níquel. As mesmas amostras foram submetidas à espectroscopia Mössbauer usando o aparelho CMTE modelo MA250, com fonte de ${ }^{57} \mathrm{Co} / \mathrm{RH}$.

Os experimentos de adsorção e dessorção com $\mathrm{Cd}$ e $\mathrm{Pb}$ em sistemas monoelementares foram feitos em valores de $\mathrm{pH}$ 5,5 e 6,5, e no valor de $\mathrm{pH}$ natural da suspensão dos adsorventes testados, os quais se encontram descritos na Tabela 1.

Tabela 1. Identificação e descrição dos materiais adsorventes estudados

\begin{tabular}{|c|c|}
\hline Identificação & Descrição \\
\hline L.F. nat & Lama da mineração de ferro in natura \\
\hline L.A. nat & Lama da mineração de alumínio in natura \\
\hline L.A. 0 P & $\begin{array}{l}\text { Lama da mineração de alumínio in natura tratada } \\
\qquad \text { com } 0 \mathrm{mg} \mathrm{kg}^{-1} \text { de fosfato }\end{array}$ \\
\hline L.A. $1.000 \mathrm{P}$ & $\begin{array}{l}\text { Lama da mineração de alumínio in natura tratada } \\
\qquad \text { com } 1.000 \mathrm{mg} \mathrm{kg}^{-1} \text { de fosfato }\end{array}$ \\
\hline L.A. 2.000 P & $\begin{array}{l}\text { Lama da mineração de alumínio in natura tratada } \\
\qquad \text { com } 2.000 \mathrm{mg} \mathrm{kg}^{-1} \text { de fosfato }\end{array}$ \\
\hline L.A. 4.000 P & $\begin{array}{l}\text { Lama da mineração de alumínio in natura tratada } \\
\text { com } 4.000 \mathrm{mg} \mathrm{kg}^{-1} \text { de fosfato }\end{array}$ \\
\hline L.A. $105 \mathrm{~T}$ & $\begin{array}{l}\text { Lama da mineração de alumínio in natura tratada } \\
\qquad \text { a } 105^{\circ} \mathrm{C}\end{array}$ \\
\hline L.A. $300 \mathrm{~T}$ & $\begin{array}{l}\text { Lama da mineração de alumínio in natura tratada } \\
\qquad \text { a } 300{ }^{\circ} \mathrm{C}\end{array}$ \\
\hline L.A. $600 \mathrm{~T}$ & $\begin{array}{l}\text { Lama da mineração de alumínio in natura tratada } \\
\qquad \text { a } 600{ }^{\circ} \mathrm{C}\end{array}$ \\
\hline
\end{tabular}

Os testes de adsorção/dessorção constaram, basicamente, de três etapas: ajuste do pH, adsorção e dessorção.

Na primeira etapa, ajuste do $\mathrm{pH}$, pesaram-se, em triplicata, 0,3 $\mathrm{g}$ dos adsorventes, os quais foram suspensos em $20 \mathrm{~mL}$ de $\mathrm{Ca}\left(\mathrm{NO}_{3}\right)_{2}$ $0,01 \mathrm{~mol} \mathrm{~L}^{-1}$ para ajuste do $\mathrm{pH}$ (relação adsorvente:solução de 1:67). Para isso, utilizaram-se quantidades predeterminadas de $\mathrm{HNO}_{3}$ a 1 mol L $\mathrm{L}^{-1}$ e solução saturada de $\mathrm{Ca}(\mathrm{OH})_{2}$, as quais foram calculadas por equações de regressão ajustadas às curvas de titulação obtidas em experimento anterior. Após a adição do ácido ou da base, alternaramse $12 \mathrm{~h}$ de repouso e $12 \mathrm{~h}$ de agitação, com as leituras de $\mathrm{pH}$ sendo feitas diariamente até se atingir a estabilidade. Considerou-se o $\mathrm{pH}$ estável quando a variação da leitura foi menor que 0,2 unidades, o que ocorreu em torno de 5 dias. Todos os experimentos foram conduzidos em $\mathrm{Ca}\left(\mathrm{NO}_{3}\right)_{2}$, pois o $\mathrm{Ca}^{2+}$ tende a ser o principal cátion básico no 
complexo sortivo dos solos e o $\mathrm{NO}_{3}$ - possui pequena capacidade de formar par iônico com metais em solução. ${ }^{20}$

Na segunda etapa, adsorção, logo após a estabilização do pH na solução com o adsorvente, adicionaram-se $10 \mathrm{~mL}$ da solução de $\mathrm{Cd}\left(\mathrm{NO}_{3}\right)_{2}$ e $\mathrm{Pb}\left(\mathrm{NO}_{3}\right)_{2}$ a $1 \mathrm{mmol} \mathrm{L}$ (relação adsorvente:solução final de 1:100). As amostras foram mantidas nos tubos de centrífuga por mais $72 \mathrm{~h}$, alternando-se $12 \mathrm{~h}$ de agitação e $12 \mathrm{~h}$ de repouso, para que se processassem as reações. Em seguida, as amostras foram centrifugadas durante $20 \mathrm{~min}$ a $3.000 \mathrm{rpm}$ e o sobrenadante coletado para leitura da quantidade remanescente em solução. A quantidade adsorvida do metal foi calculada pela diferença entre a adicionada e a remanescente na solução de equilíbrio.

Na terceira etapa, dessorção, ao resíduo remanescente da adsorção nos tubos de centrífuga, adicionaram-se $30 \mathrm{~mL}$ de $\mathrm{Ca}\left(\mathrm{NO}_{3}\right)_{2}$,, $01 \mathrm{~mol}$ $\mathrm{L}^{-1}$, a fim de promover a dessorção dos elementos-traço adsorvidos. As amostras foram mantidas nos tubos de centrífuga por mais $72 \mathrm{~h}$, alternando-se $12 \mathrm{~h}$ de agitação e $12 \mathrm{~h}$ de repouso. Em seguida, foram centrifugadas, durante $20 \mathrm{~min}$ a $3.000 \mathrm{rpm}$, sendo o sobrenadante coletado para leitura da quantidade dessorvida. A quantidade dessorvida foi calculada descontando-se a concentração do metal retido na solução de equilíbrio proveniente da adsorção prévia. As leituras das quantidades adsorvidas e dessorvidas foram feitas por espectrofotometria de absorção atômica de chama e com forno de grafite para as leituras na faixa de concentração em $\mathrm{mg} \mathrm{L}^{-1}$ e $\mu \mathrm{g} \mathrm{L} \mathrm{L}^{-1}$, respectivamente.

\section{RESULTADOS E DISCUSSÃO}

Na tentativa de se usar a lama da mineração de alumínio in natura como um substrato, ela foi submetida à análise padrão de fertilidade do solo. Como se pode observar pelos resultados descritos na Tabela 2 , este material apresenta algumas características que impossibilitam

Tabela 2. Atributos químicos da lama da mineração de alumínio in natura

\begin{tabular}{|c|c|c|}
\hline Atributo $^{(1)}$ & Unidade & Valores médios \\
\hline pH em água $(1: 2,5)$ & - & $10,0 \pm 0$ \\
\hline P (Mehlich-1) & $\mathrm{mg} \mathrm{dm} \mathrm{m}^{-3}$ & $0,9 \pm 0$ \\
\hline K (Mehlich-1) & $\mathrm{mg} \mathrm{dm} \mathrm{m}^{-3}$ & $193,5 \pm 3,5$ \\
\hline $\mathrm{Na}$ (Mehlich-1) & $\mathrm{mg} \mathrm{dm}{ }^{-3}$ & $27526,4 \pm 624,5$ \\
\hline $\mathrm{Ca}^{+2}\left(\mathrm{KCl} 1 \mathrm{~mol} \mathrm{~L}^{-1}\right)$ & $\mathrm{cmol}_{\mathrm{c}} \mathrm{dm}^{-3}$ & $0,3 \pm 0$ \\
\hline $\mathrm{Mg}^{+2}\left(\mathrm{KCl} 1 \mathrm{~mol} \mathrm{~L}^{-1}\right)$ & $\mathrm{cmol}_{\mathrm{c}} \mathrm{dm}^{-3}$ & $0,1 \pm 0$ \\
\hline $\mathrm{Al}^{+3}\left(\mathrm{KCl} 1 \mathrm{~mol} \mathrm{~L}^{-1}\right)$ & $\mathrm{cmol}_{\mathrm{c}} \mathrm{dm}^{-3}$ & $0 \pm 0$ \\
\hline Acidez potencial $(\mathrm{H}+\mathrm{Al})(\mathrm{SMP})$ & $\mathrm{cmol}_{\mathrm{c}} \mathrm{dm}^{-3}$ & $0,2 \pm 0$ \\
\hline Soma de bases & $\mathrm{cmol}_{\mathrm{c}} \mathrm{dm}^{-3}$ & $120,6 \pm 2,7$ \\
\hline CTC efetiva & $\mathrm{cmol}_{\mathrm{c}} \mathrm{dm}^{-3}$ & $120,6 \pm 2,7$ \\
\hline CTC a pH 7,0 & $\mathrm{cmol}_{\mathrm{c}} \mathrm{dm}^{-3}$ & $120,8 \pm 2,7$ \\
\hline Saturação por bases & $\%$ & $99,8 \pm 0$ \\
\hline Saturação por Al & $\%$ & $0 \pm 0$ \\
\hline Saturação por Na & $\%$ & $99,3 \pm 0$ \\
\hline$P$ remanescente & $\mathrm{mg} \mathrm{L}^{-1}$ & $3,7 \pm 0,3$ \\
\hline Zn (Mehlich-1) & $\mathrm{mg} \mathrm{dm}^{-3}$ & $0,1 \pm 0$ \\
\hline Fe (Mehlich-1) & $\mathrm{mg} \mathrm{dm}{ }^{-3}$ & $2,3 \pm 1,8$ \\
\hline Mn (Mehlich-1) & $\mathrm{mg} \mathrm{dm}{ }^{-3}$ & $1,0 \pm 0,4$ \\
\hline $\mathrm{Cu}$ (Mehlich-1) & $\mathrm{mg} \mathrm{dm}^{-3}$ & $0,3 \pm 0,1$ \\
\hline
\end{tabular}

(1) Conforme ref. 16 a sua utilização como substrato na sua forma in natura. Alguns atributos químicos, tais como, alto $\mathrm{pH}$ e alta concentração de sódio $(\mathrm{Na})$, não permitiriam o desenvolvimento da grande maioria das plantas cultivadas. Além disso, é um material pobre em nutrientes, o que limita ainda mais o seu uso como substrato.

Estudos comprovaram que o $\mathrm{pH}$ da lama pode ser reduzido, pela irrigação com água do mar, para aproximadamente 8. Uma outra vantagem observada foi a substituição de $\mathrm{Na}$ por $\mathrm{Ca}, \mathrm{Mg}$ e K, porém, apesar da salinidade ser reduzida, o material permaneceu ainda com uma concentração salina muito elevada. ${ }^{21}$ Esses resultados corroboram o presente trabalho, pois mesmo após ter sido tratada, a lama apresentou restrições para o crescimento da maioria das espécies de plantas, no entanto, isso não inviabiliza seu potencial de uso como adsorvente e amenizante de solos contaminados com elementos-traço.

Com base no resultado do ataque sulfúrico, o teor de óxidos da lama da mineração de alumínio in natura foi de $15,76 \%$ de $\mathrm{SiO}_{2}$, $31,68 \%$ de $\mathrm{Al}_{2} \mathrm{O}_{3}, 15,53 \%$ de $\mathrm{Fe}_{2} \mathrm{O}_{3}, 3,06 \%$ de $\mathrm{TiO}_{2}, 0,20 \%$ de $\mathrm{P}_{2} \mathrm{O}_{5}$ e 3,20\% da razão molar de $\mathrm{Al}_{2} \mathrm{O}_{3} / \mathrm{Fe}_{2} \mathrm{O}_{3}$. Observa-se que o material apresentou aproximadamente o dobro da concentração de $\mathrm{Al}_{2} \mathrm{O}_{3}$, quando comparado com $\mathrm{Fe}_{2} \mathrm{O}_{3}$ e $\mathrm{SiO}_{2}$. Estudos efetuados com a lama da mineração de alumínio reportaram o seguinte: $9,58-15,74 \%$ de $\mathrm{SiO}_{2}, 17,28-20,39 \%$ de $\mathrm{Al}_{2} \mathrm{O}_{3}, 30,45-38,80 \%$ de $\mathrm{Fe}_{2} \mathrm{O}_{3}, 4,98-18,80 \%$ de $\mathrm{TiO}_{2}$ e $0,20-0,50 \%$ de $\mathrm{P}_{2} \mathrm{O}_{5}{ }^{3,8,10,22}$ Nota-se por estes resultados, que o material deste estudo se diferencia daquele descrito na literatura internacional, basicamente pelo seu teor de $\mathrm{Fe}_{2} \mathrm{O}_{3}$. Pelo fato de não se conhecer o processo de determinação dos óxidos descritos na literatura internacional, a comparação, às vezes, se torna um tanto quanto subjetiva.

As quantidades semitotais de $\mathrm{Cd}$ e $\mathrm{Pb}$ dos adsorventes encontramse na Tabela 3. Nota-se que a lama da mineração de ferro na sua forma in natura apresenta valores para $\mathrm{Cd}$ e $\mathrm{Pb}$ bem menores que os da lama da mineração de alumínio in natura. Em geral, as quantidades de $\mathrm{Cd}$ e $\mathrm{Pb}$ presentes na lama não inviabilizam seu uso como adsorvente. Valores reportados na literatura ${ }^{6,8,10}$ variam de 8,2 a 23,0 $\mathrm{mg} \mathrm{kg}^{-1}$ para $\mathrm{Cd}$ e de 163 a $175 \mathrm{mg} \mathrm{kg}^{-1}$ para $\mathrm{Pb}$, indicando que as concentrações encontradas neste estudo são bem menores. A comparação entre os valores torna-se um tanto quanto subjetiva, pois as metodologias usadas pelos autores foram diferentes, apesar de ter sido evidenciada, pela literatura, uma grande variabilidade existente no material.

Tendo-se em mente que as cargas superficiais dos adsorventes desempenham um papel muito importante nas reações de troca que

Tabela 3. Teores semitotais de $\mathrm{Cd}$ e $\mathrm{Pb}$ nos materiais adsorventes estudados

\begin{tabular}{lcc}
\hline Adsorvente $^{(1)}$ & $\begin{array}{c}\mathrm{Cd}^{(2)} \\
\mathrm{Pb}^{(2)}\end{array}$ & $\begin{array}{c}\text {------ } \mathrm{mg} \mathrm{kg}^{-1}---- \\
\text { L. F. nat }\end{array}$ \\
L. A. nat & 0,53 & 6,77 \\
L. A. 0 P & 19,92 & 69,39 \\
L. A. 1000 P & 29,84 & 82,29 \\
L. A. 2000 P & 26,46 & 80,12 \\
L. A. 4000 P & 23,12 & 78,77 \\
L. A. 105 T & 30,14 & 81,42 \\
L. A. 300 T & 40,61 & 74,19 \\
L. A. 600 T & 35,90 & 71,88 \\
\hline
\end{tabular}

${ }^{(1)} \mathrm{A}$ identificação dos materiais adsorventes estudados encontra-se na Tabela 1. ${ }^{(2)}$ Determinações das quantidades semitotais de metais pelo método USEPA 3051A. 
ocorrem nos solos, foi determinado o ponto de carga zero (PCZ) para se ter uma referência da carga líquida da superfície dos adsorventes para um determinado valor de $\mathrm{pH}$ (Tabela 4). O adsorvente L.F. nat apresentou maior valor de PCZ quando comparado ao L.A. nat. O pré-tratamento com ácido fosfórico reduziu os valores de PCZ dos adsorventes, provavelmente, devido à adsorção do fosfato, resultando em maior balanço líquido de cargas negativas nas suas superfícies.

Tabela 4. Ponto de carga zero dos adsorventes

\begin{tabular}{lc}
\hline Adsorvente $^{(1)}$ & $\mathrm{PCZ}^{(2)}$ \\
\hline L. F. nat & 6,36 \\
L. A. nat & 5,95 \\
L. A. $0 \mathrm{P}$ & $4,78 \pm 0,16$ \\
L. A. $1000 \mathrm{P}$ & $4,28 \pm 0,15$ \\
L. A. $2000 \mathrm{P}$ & $4,34 \pm 0,12$ \\
L. A. $4000 \mathrm{P}$ & $4,72 \pm 0,02$ \\
L. A. $105 \mathrm{~T}$ & $5,30 \pm 0,28$ \\
L. A. $300 \mathrm{~T}$ & $6,11 \pm 0,35$ \\
L. A. $600 \mathrm{~T}$ & $5,52 \pm 0,05$ \\
\hline
\end{tabular}

(1) A identificação dos materiais adsorventes estudados encontra-se na Tabela 1. ${ }^{(2)} \mathrm{PCZ}$ - Ponto de carga zero.

O pré-tratamento térmico provocou mudanças nas superfícies dos adsorventes, devido a perdas de determinados elementos em função do aquecimento, o que, conseqüentemente, altera o balanço de cargas da superfície. O PCZ é um importante parâmetro a ser considerado para estes adsorventes, devido a sua constituição oxídica. Os óxidos apresentam caráter anfótero devido às cargas dependentes do $\mathrm{pH}$. Eles podem desenvolver cargas negativas quando o $\mathrm{pH}$ do meio for maior que o PCZ e cargas positivas quando o valor de $\mathrm{pH}$ estiver abaixo do PCZ. ${ }^{14,23}$

Dentre os vários constituintes da lama da mineração de alumínio, a sílica, com ponto de carga zero (PCZ) próximo de 2,3, contribui para abaixar o PCZ final da lama, enquanto que o $\mathrm{Fe}_{2} \mathrm{O}_{3}$, com PCZ próximo de 8,6 , contribui para aumentar o PCZ final da lama, sendo reportado na literatura um valor igual a $3,2 .^{3}$

No que diz respeito à caracterização mineralógica, na Figura 1 podem-se verificar os difratogramas de raios- $\mathrm{X}$ dos adsorventes. $\mathrm{O}$ difratograma do adsorvente L.F. nat apresentou picos bem evidentes, indicando a presença de hematita, goethita e quartzo. Já os adsorventes L.A. nat; L.A. 105 T e L.A. $300 \mathrm{~T}$ apresentaram picos indicando a presença de hematita, goethita, maghemita, quartzo e gibbsita.

Com o pré-tratamento da lama da mineração de alumínio a 600 ${ }^{\circ} \mathrm{C}$ (L.A. $600 \mathrm{~T}$ ), os picos da gibbsita nos espaçamentos 0,433 e 0,483 $\mathrm{nm}$ e o pico da goethita no espaçamento $0,416 \mathrm{~nm}$ desapareceram, indicando o efeito do pré-tratamento térmico em alterar a composição mineralógica do material adsorvente. A hematita pode derivar da desidroxilação da goethita a temperaturas abaixo de $600{ }^{\circ} \mathrm{C}$ e, durante a desidroxilação, as ligações $\mathrm{OH}$ são substituídas por ligações oxigênio e desenvolve-se o compartilhamento de faces entre octaedros. ${ }^{13}$

Determinações feitas na lama da mineração de alumínio com o uso da difração de raios-X evidenciaram a presença de muscovita, hematita, goethita, quartzo, calcita, anatásio, sodalita, gibbsita e maghemita, ${ }^{24}$ enquanto que outros trabalhos ${ }^{8,10}$ encontraram, como principais constituintes, alumínio na forma de boehmita e gibbsita, hematita, cancrinita, ilmenita, rutilo e quartzo.

Outros trabalhos envolvendo a lama da mineração de alumínio retratam que sua composição mineralógica é muito variável consti-

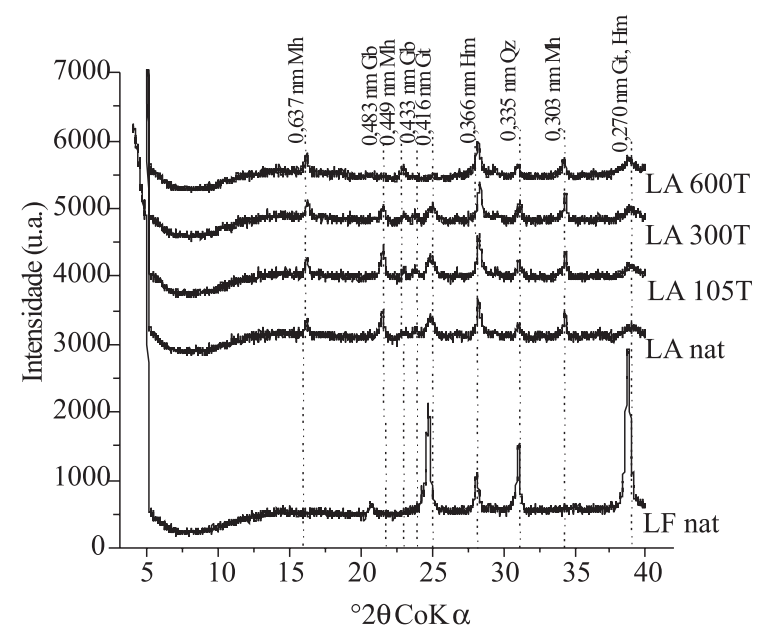

Figura 1. Difratogramas de raios-X dos materiais adsorventes L.F. nat, L.A. nat, L.A. 105 T, L.A. 300 T e L.A. 600 T, cujas descrições encontram-se na Tabela 1. Os números acima dos picos representam os espaçamentos em nm com seus respectivos minerais: $M h$-maghemita, Gb-gibbsita, Gt-goethita, $H m$ - hematita e $Q z$ - quartzo

tuindo-se de: 10-35\% de hematita, 10-30\% de alumo-goethita, 4-40\% de sodalita, 2-20\% de aluminato tricálcico, 1,5-15\% de anatásio/ rutilo, $1-10 \%$ de calcita, $0-30 \%$ de quartzo, 0-20\% de boehmita, $0-5 \%$ de gibbsita, $0-5 \%$ de caulinita, $0-15 \%$ de muscovita, $4,60 \%$ de cancrinita e $2,50 \%$ de diásporo, sendo a bayerita também presente em menores quantidades. ${ }^{3,22,25}$ Também verificaram, por microscopia eletrônica de varredura, que a lama ativada apresentou porosidade e textura da superfície similares às de silicatos de alumínio contendo impurezas tais como ferro. ${ }^{3}$

Os espectros obtidos pela espectroscopia Mössbauer a temperatura ambiente são apresentados na Figura 2. Analisando-se inicialmente os espectros Mössbauer das amostras naturais L.A. nat e L.F. nat, pode-se constatar que a lama L.A. nat apresenta-se com cristais de menor tamanho de partícula quando comparada à lama L.F. nat, conforme indicado pelo acentuado dupleto central observado na Figura 2a. Esse resultado corrobora os dados de difração de raios-X onde ficou evidente o maior grau de cristalinidade do L.F. nat. A literatura sugere que a razão para tal efeito seja a substituição isomórfica de ferro por alumínio na estrutura das fases de ferro. ${ }^{23}$

$\mathrm{O}$ valor de campo hiperfino, $\mathrm{B}_{\mathrm{hf}}$, de 50,4 tesla obtido de um ajuste do espectro da Figura 2 a sugere a presença de um sexteto referente à fase maghemita $\left(\gamma \mathrm{Fe}_{2} \mathrm{O}_{3}\right)$, sendo que as fases hematita $\left(\alpha \mathrm{Fe}_{2} \mathrm{O}_{3}\right) \mathrm{e}$ goethita $(\alpha \mathrm{FeOOH})$ identificadas na difração de raios-X podem estar presentes como partículas muito pequenas dando origem ao dupleto central. Por outro lado, a amostra L.F. nat pode ser ajustada com três sextetos de campo hiperfino de 51,8, 39,0 e 35,6 tesla. $O$ valor mais elevado de $B_{h f}$ refere-se à fase hematita e os valores de 39 e 35,6 são referentes à goethita, sendo que existe possivelmente uma fase mais substituída por alumínio resultando no valor de campo de 35,6 tesla. ${ }^{26}$ Pode-se observar ainda um dupleto central pouco pronunciado, podendo ser novamente a goethita altamente substituída por $\mathrm{Al}$ ou ainda ferro presente em silicatos, que pode ser comprovado com análises Mössbauer a baixa temperatura, como a do nitrogênio líquido.

Com relação aos espectros Mössbauer da Figura 2b, pode-se observar que os tratamentos a 105 e $300{ }^{\circ} \mathrm{C}$ não modificaram significativamente as fases de ferro presentes, sendo detectado apenas o campo hiperfino de 50,4 tesla referente à maghemita, anteriormente detectada no adsorvente L.A. nat. Por outro lado, a amostra tratada a $600{ }^{\circ} \mathrm{C}$ (L.A. 600 T) apresentou uma brusca diminuição do dupleto central com o aparecimento de 3 sextetos com valores de campo 


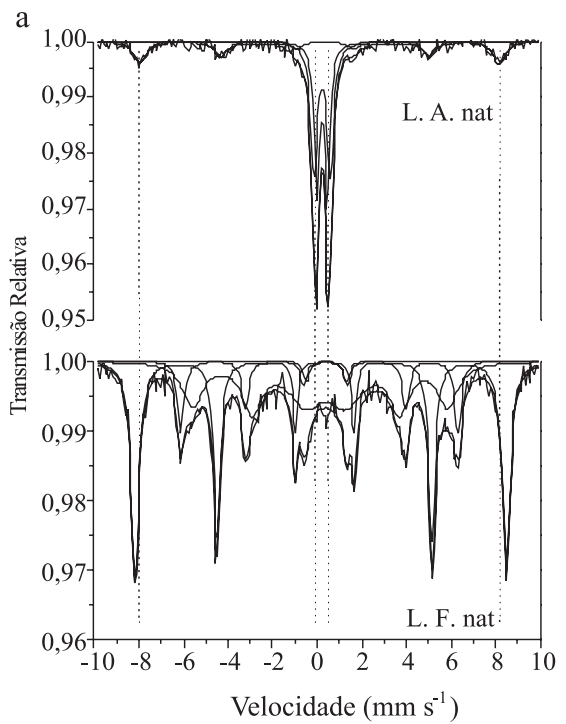

b

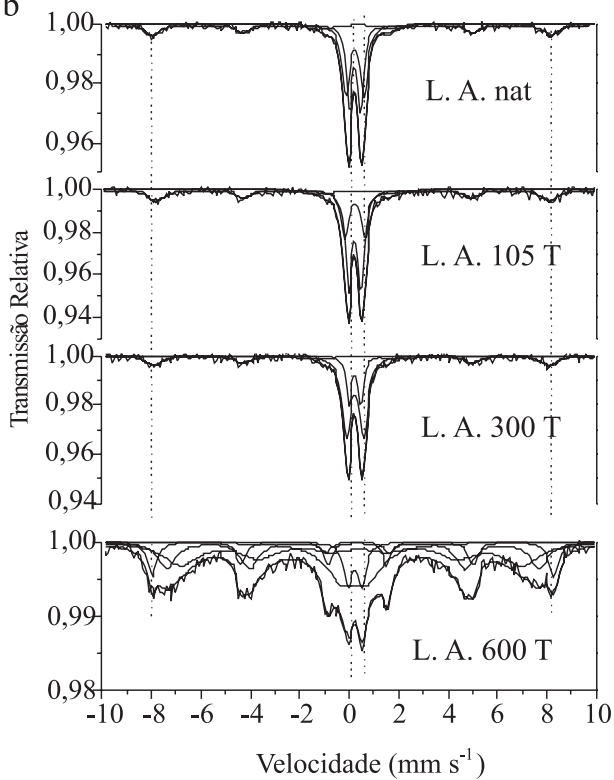

Figura 2. Espectros Mössbauer a temperatura ambiente dos materiais adsorventes L.F. nat e L.A. nat (a); L.A. nat, L.A. 105 T, L.A. 300 T e L.A. 600 $T(b)$, cujas descrições encontram-se na Tabela 1

hiperfino de 51,4, 46,8 e 42,9 tesla, sendo relacionados à presença de hematita e maghemita, respectivamente. É interessante observar ainda que o aparecimento dos sextetos sugere um aumento no tamanho de partícula pela agregação dos materiais com o tratamento térmico, o que poderá influenciar o processo de adsorção dos íons metálicos em solução.

Esses resultados estão de acordo com aqueles obtidos na difratometria de raios- $\mathrm{X}$, onde ficou evidenciada a desidroxilacão da goethita para formação de hematita, de acordo com a Equação 1.

$$
\mathrm{FeOOH} \underset{\mathrm{T}=600^{\circ} \mathrm{C}}{\longrightarrow} \mathrm{Fe}_{2} \mathrm{O}_{3}+\mathrm{H}_{2} \mathrm{O}
$$

Pode-se notar, em função dos resultados obtidos neste estudo e daqueles descritos na literatura, que o material em estudo apresenta uma composição muito variada com relação aos diversos atributos analisados, o que pode ser atribuído à matéria-prima bauxita e a variações apresentadas no método de extração do alumínio. Essa variação confirma a necessidade de uma ampla caracterização dos materiais de origens distintas (e.g., local, material de origem, tipo de processamento, entre outros) a serem reutilizados como adsorventes ou amenizantes.

As frações percentuais adsorvidas de $\mathrm{Cd}$ e $\mathrm{Pb}$ nos materiais adsorventes encontram-se na Figura 3. Pode-se observar que a elevação do $\mathrm{pH}$ proporcionou um aumento nas frações percentuais de $\mathrm{Cd} \mathrm{e}$ $\mathrm{Pb}$ adsorvidas. Resultados semelhantes foram obtidos em trabalhos envolvendo a adsorção de $\mathrm{Cd}$ e $\mathrm{Pb}$ na própria lama da mineração de alumínio e em Latossolos (material com característica oxídica, i.e., semelhante aos adsorventes usados no presente estudo). ${ }^{12,27-29}$

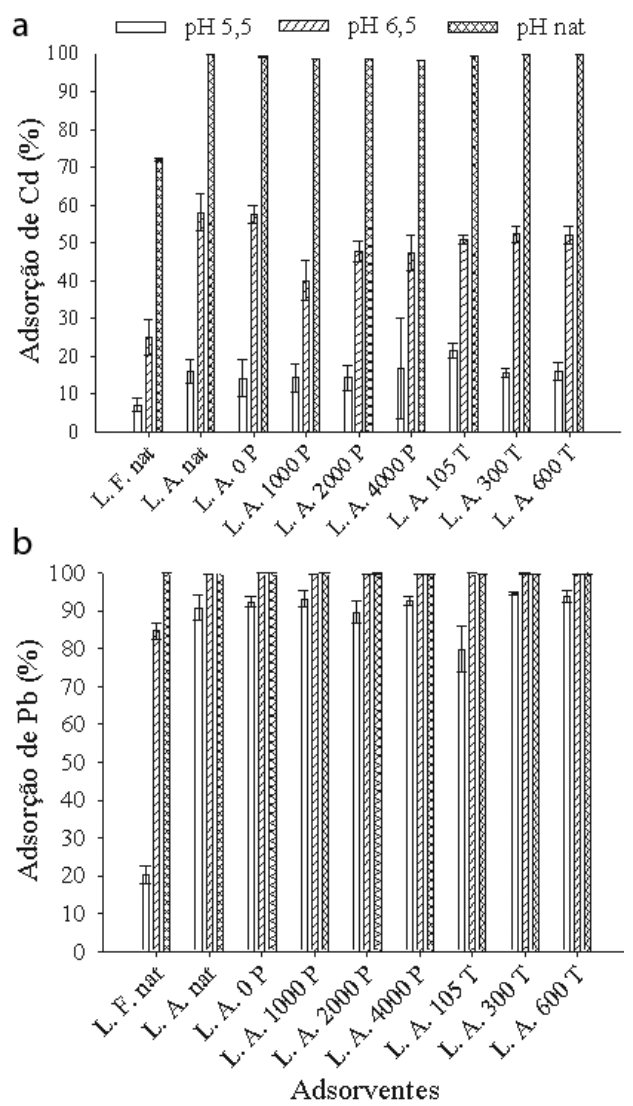

Figura 3. Fração percentual de $C d(a)$ e de $P b(b)$ adsorvido [Metal ${ }_{\sigma_{c a d s}}=$ $\left(\right.$ Metal $_{\text {adsorvido }} /$ Metal $\left._{\text {adicionado }}\right) \times 100$ ] na L.F. nat, L.A. nat, L.A. O P, L.A. 1.000 P, L.A. 2.000 P, L.A. 4.000 P, L.A. 105 T, L.A. 300 T e L.A. 600 T. A descrição dos materiais adsorventes estudados encontra-se na Tabela 1. Os valores de $\mathrm{pH}$ da solução de equilíbrio $\left[\mathrm{Ca}\left(\mathrm{NO}_{3}\right)_{2} 0,01 \mathrm{~mol} \mathrm{~L}^{-1}\right]$ foram: $\mathrm{pH} 5,5 \pm 0,0 ; 6,5 \pm 0,1$ e natural (L.F. nat $=8,0 \pm 0,0 ;$ L.A. nat $=9,5 \pm 0,0 ; L . A . O P=10,0 \pm 0,0 ;$ L.A. $1.000 P=9,8 \pm 0,0 ;$ L.A. $2.000 P=9,8 \pm 0,0 ;$ L.A. $4.000 P=9,6 \pm 0,0 ;$ L.A. 105 $T=10,4 \pm 0,0 ;$ L.A. $300 T=10,5 \pm 0,0 ;$ L.A. $600 T=10,5 \pm 0,0)$. Quantidade total adicionada de cada metal na adsorção $=33,33 \mathrm{mmol} \mathrm{kg}^{-1} \mathrm{com}$ relação adsorvente:solução final de 1:100

Os incrementos obtidos na adsorção com o aumento do $\mathrm{pH}$ foram bastante evidentes para o Cd (Figura 3a) e para o $\mathrm{Pb}$ (Figura $3 b)$. Vale ressaltar que, em $\mathrm{pH} 6,5$, todos os materiais adsorventes, provavelmente, apresentam um balanço de carga líquida negativo, pelo fato do $\mathrm{pH}$ estar acima do $\mathrm{PCZ}$, o que favorece a adsorção de metais catiônicos. Pelo fato de ter ocorrido adsorção a pH 5,5 e alguns materiais apresentarem PCZ muito próximo ou abaixo deste valor, pode-se concluir que, provavelmente, não está ocorrendo somente atração eletrostática, mas também adsorção via complexos de esfera interna. Estes resultados evidenciam uma maior afinidade dos adsorventes pelo $\mathrm{Pb}$, quando comparado ao $\mathrm{Cd}$.

A diferença no comportamento desses dois elementos não ocorre somente em função das superfícies adsorventes conforme discutido 
em experimentos de adsorção em solos oxídicos, mas também devido às diferenças nas configurações eletrônicas dos próprios metais. ${ }^{30}$ $\mathrm{O} \mathrm{Pb}$ apresenta elétrons desemparelhados em sua camada externa, que facilitam as ligações e o compartilhamento dos mesmos. Além do mais, os espaços vazios nos orbitais desse elemento permitem a formação de complexos, o que não é verificado para o $\mathrm{Cd}$.

Estudos envolvendo a influência da força iônica da solução de equilíbrio na adsorção de $\mathrm{Cd}$ e $\mathrm{Pb}$ retrataram que $\mathrm{o} \mathrm{Pb}$ é adsorvido predominantemente como complexo de esfera interna, não fazendo parte do complexo de troca e que a adsorção de Cd é típica de reações nas quais há a predominância de formação de complexos de esfera externa. ${ }^{31,32}$

$\mathrm{O}$ adsorvente L.A. nat ou pré-tratado mostrou-se mais promissor em adsorver $\mathrm{Cd}$ e $\mathrm{Pb}$, quando comparado com o L.F. nat. Os pré-tratamentos com ácido fosfórico e térmico não proporcionaram aumento nas frações percentuais adsorvidas de $\mathrm{Cd}$ e $\mathrm{Pb}$. O pré-tratamento com fósforo de amostras de Latossolos com balanços positivos de cargas em subsuperfície apresentou resultados promissores. ${ }^{15}$ Quando se tem em mente a utilização deste material como adsorvente, o fato de não ser preciso um pré-tratamento torna estes resultados interessantes, pois viabiliza as operações e os custos envolvidos numa eventual preparação do material para ser usado como amenizante.

De modo geral, pode-se verificar a superioridade da L.A. nat quando comparada com a L.F. nat com relação à capacidade de adsorção. Trabalhos utilizando a lama da mineração de alumínio como adsorvente de $\mathrm{Cd}$ e $\mathrm{Zn}$ de soluções aquosas retratam a eficiência deste material como adsorvente. ${ }^{3}$ Foi verificado também que a concentração de metais na solução dos solos e os fluxos dos mesmos foram diminuídos pela aplicação de $2 \%$ de lama vermelha. ${ }^{6}$

Vale ressaltar que, devido ao fato desses adsorventes apresentarem reação alcalina, não só a adsorção, mas também a precipitação dos elementos-traço, devido à formação de carbonatos e hidróxidos, constitui-se numa forma de redução da concentração destes elementos em solução. Estudo da redução da disponibilidade de $\mathrm{Cd}, \mathrm{Zn}$ e $\mathrm{Cu}$ em solo sugere que o mecanismo de ação do calcário e da beringita é, provavelmente, relacionado ao aumento da adsorção de metal e à precipitação de hidróxido e carbonato de metal a um alto valor de pH. No caso da lama, a combinação de mecanismos dependentes e independentes do $\mathrm{pH}$ pode ser responsável pela fixação do metal como, por exemplo, possível difusão para a fase sólida ou migração para dentro de microporos. ${ }^{12}$

Além de se constituírem em bons adsorventes, os materiais testados neste trabalho apresentaram também uma boa capacidade de manterem os elementos-traço adsorvidos (Figuras 4). As frações percentuais dessorvidas de $\mathrm{Cd}$ (Figura $4 \mathrm{a}$ ) e $\mathrm{Pb}$ (Figura 4b) foram reduzidas com o aumento do $\mathrm{pH}$. $\mathrm{O} \mathrm{Pb}$ foi menos dessorvido que o Cd para todos os valores de $\mathrm{pH}$ analisados. Contudo, a dessorção de $\mathrm{Cd}$ e de $\mathrm{Pb}$ foi maior quando a adsorção ocorreu em valores de $\mathrm{pH}$ mais baixos.

Os adsorventes tenderam a aumentar a percentagem de Cd dessorvida a $\mathrm{pH}$ natural na seguinte ordem crescente: L.A. nat, L.A. 0 P, L.A. 1.000 P, L.A. 2.000 P e L.A. 4.000 P (Figura 4a). O contrário foi verificado para o pré-tratamento térmico, com valores maiores para L.A. 105 T, seguido do L.A. 300 T e L.A. 600 T. Este comportamento inverso pode ser atribuído ao efeito do pré-tratamento em alterar a superfície dos adsorventes. No caso do pré-tratamento com fósforo, o mesmo altera o balanço de cargas da superfície do adsorvente, fazendo com que ocorra a adsorção não específica ou o complexo de esfera externa, proporcionando maior facilidade de deslocamento dos elementos para a solução de equilíbrio. No caso do pré-tratamento térmico, ocorrem modificações na estrutura do material adsorvente e, neste caso, o novo rearranjo parece manter o $\mathrm{Pb}$ mais fortemente adsorvido, propiciando a adsorção via complexo de esfera interna ou

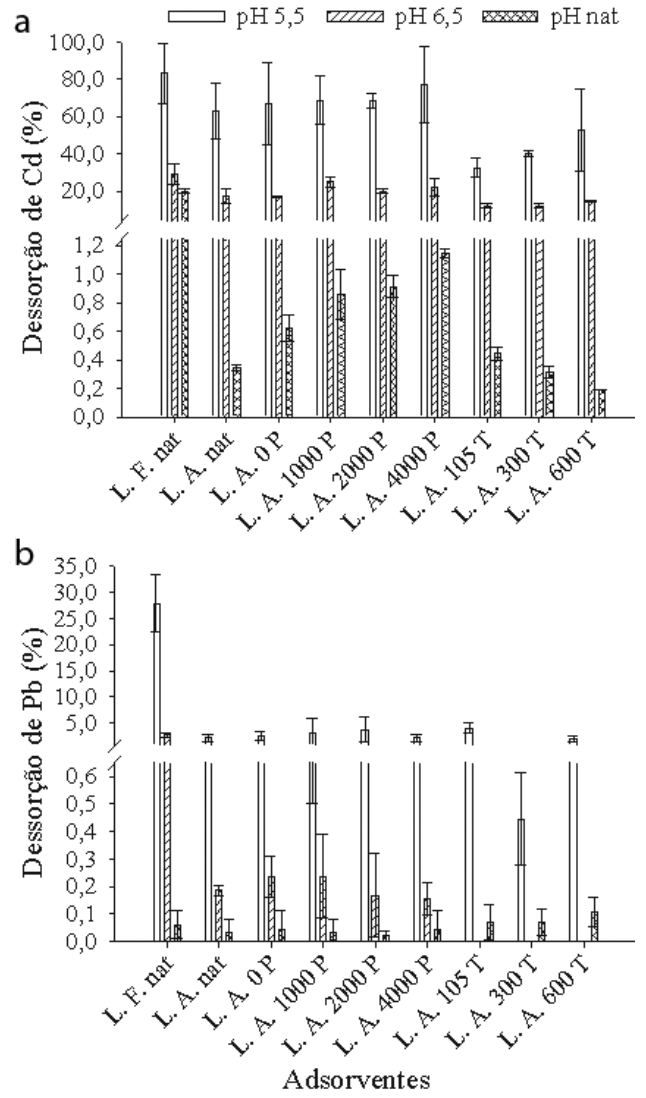

Figura 4. Fração percentual de $C d(a)$ e de $\mathrm{Pb}(\mathrm{b})$ dessorvido [Metal $_{\% \text { des }}=$ $\left(\right.$ Metal $_{\text {dessorvido }} /$ Metal $\left._{\text {adsorvido }}\right) \times 100$ ] na L.F. nat, L.A. nat, L.A. O P, L.A. 1.000 P, L.A. 2.000 P, L.A. 4.000 P, L.A. 105 T, L.A. 300 T e L.A. 600 T. A descrição dos materiais adsorventes estudados encontra-se na Tabela 1. Os valores de $p H$ da solução de equilíbrio [ $\mathrm{Ca}\left(\mathrm{NO}_{3}\right)_{2}$ 0,01 mol L-1] foram: pH 5,5 $\pm 0,0 ; 6,5 \pm 0,1$ e natural (L.F. nat $=8,0 \pm 0,0 ;$ L.A. nat $=9,5 \pm 0,0 ;$ L.A. $O P=10,0 \pm 0,0 ;$ L.A. $1.000 P=9,8 \pm 0,0 ;$ L.A. $2.000 P=9,8 \pm 0,0 ;$ L.A. $4.000 P=9,6 \pm 0,0 ;$ L.A. $105 T=10,4 \pm 0,0 ;$ L.A. $300 T=10,5 \pm 0,0 ;$ L.A. $600 T=10,5 \pm 0,0$ ). Relação adsorvente:solução final de 1:100

adsorção específica, dificultando que o elemento-traço seja deslocado de volta para a solução.

Provavelmente, uma grande fração desses metais foi realmente adsorvida e não precipitada, senão eles teriam sido deslocados para a solução de dessorção mais facilmente.

Considerando, principalmente, os valores de $\mathrm{pH}$ natural dos materiais adsorventes em que ocorreu adsorção e dessorção $(8,0 \pm 0,0$ a $10,5 \pm 0,0$ ), tem-se que considerar que na redução da concentração destes elementos na solução de equilíbrio pode ter ocorrido não somente a adsorção, mas também a precipitação, principalmente na forma de carbonatos e hidróxidos de $\mathrm{Cd}$ e $\mathrm{Pb}$. As constantes do produto de solubilidade (Kps) (e respectivas solubilidades, entre parênteses) para $\mathrm{Cd}$ e $\mathrm{Pb}$ na forma de hidróxido e carbonato, são $\mathrm{Cd}(\mathrm{OH})_{2} 7,20 \times 10^{-15}\left(1,22 \times 10^{-5} \mathrm{~mol} \mathrm{~L}^{-1}\right) ; \mathrm{CdCO}_{3} 1,00 \times 10^{-12}(1,00$ x $\left.10^{-6} \mathrm{~mol} \mathrm{~L}^{-1}\right) ; \mathrm{Pb}(\mathrm{OH})_{2} 1,43 \times 10^{-20}\left(1,53 \times 10^{-7} \mathrm{~mol} \mathrm{~L}^{-1}\right)$ e $\mathrm{PbCO}_{3}$ $7,40 \times 10^{-14}\left(2,72 \times 10^{-7} \mathrm{~mol} \mathrm{~L}^{-1}\right)$. $^{33}$

A avaliação do $\mathrm{pH}$ e da capacidade de adsorção de metais na lama da mineração de alumínio tratada com água do mar evidenciou que houve redução da alcalinidade para valores seguros, mantendo suas propriedades de adsorver metal. Conforme observado pelos autores, os resultados obtidos na dessorção parecem confirmar a hipótese de que os metais adsorvidos não são facilmente trocáveis e removíveis. ${ }^{2}$ Os autores relatam, ainda, que tais resultados são encorajadores 
para o uso do subproduto para atividades de remediação e que há a necessidade de um melhor entendimento dos mecanismos de retenção de metais pelo mesmo, em vez de se assumir uma simples co-precipitação como consequiência do aumento do $\mathrm{pH}$.

\section{CONCLUSÃO}

O subproduto da indústria de alumínio in natura apresenta, como características químicas importantes, um alto $\mathrm{pH}$ e uma alta concentração de sódio.

O subproduto da indústria de alumínio in natura demonstrou ser mais promissor que a lama da mineração de ferro, como adsorvente de $\mathrm{Cd}$ e $\mathrm{Pb}$, e os pré-tratamentos com ácido fosfórico e térmico não apresentaram incrementos na adsorção desses metais que justifiquem fazê-los quanto ao uso do subproduto como adsorvente.

A elevação do $\mathrm{pH}$ proporcionou um aumento nas frações percentuais de $\mathrm{Cd}$ e $\mathrm{Pb}$ adsorvidas e os adsorventes apresentaram uma maior afinidade em adsorver $\mathrm{Pb}$.

As frações percentuais dessorvidas de $\mathrm{Cd}$ e $\mathrm{Pb}$ foram reduzidas com o aumento no $\mathrm{pH}$ e o $\mathrm{Pb}$ foi menos dessorvido que o $\mathrm{Cd}$.

\section{AGRADECIMENTOS}

À FAPEMIG e ALCOA pelo suporte financeiro e concessão de bolsas.

\section{REFERÊNCIAS}

1. http://www.dnpm.gov.br, acessada em Julho 2008.

2. Brunori, C.; Cremisini, C.; Massanisso, P.; Pinto, V.; Torricelli, L.; J. Hazard. Mater. 2005, 117, 55.

3. Gupta, V. K.; Sharma, S.; Environ. Sci. Technol. 2002, 36, 3612.

4. Zhu, C.; Luan, Z.; Wang, Y.; Shan, X.; Sep. Purif. Technol. 2007, 57, 161.

5. Lombi, E.; Zhao, F. J.; Wieshammer, G.; Zhang, G.; McGrath, S. P.; Environ. Pollut. 2002, 118, 445.

6. Lombi, E.; Zhao, F. J.; Zhang, G.; Sun, B.; Fitz, W.; Zhang, H.; McGrath, S. P.; Environ. Pollut. 2002, 118, 435.

7. Friesl, W.; Horak, O.; Wenzel, W. W.; J. Plant Nutr. Soil Sci. 2004, 167, 54.

8. Ciccu, R.; Ghiani, M.; Serci, A; Fadda, S.; Peretti, R.; Zucca, A.; Miner. Eng. 2003, 16, 187.

9. Lombi, E.; Hamon, R. E.; Wieshammer, G.; McLaughlin, M. J.; McGrath, S. P.; J. Environ. Qual. 2004, 33, 902.

10. Bertocchi, A. F.; Ghiani, M.; Peretti, R.; Zucca, A.; J. Hazard. Mater. 2006, 134, 112.

11. Lombi, E.; Hamon, R. E.; Wieshammer, G.; McLaughlin, M. J.; McGrath, S. P.; J. Environ. Qual. 2004, 33, 902.
12. Lombi, E.; Hamon, R. E.; McGrath, S. P.; McLaughlin, M. J.; Environ. Sci. Technol. 2003, 37, 979.

13. Kämpf, N.; Curi, N. Em Óxidos de ferro: indicadores de ambientes pedogenéticos e geoquímicos; Novais, R. F.; Alvarez V., V. H.; Schaefer, C. E., eds.; Sociedade Brasileira de Ciência do Solo: Viçosa, 2000.

14. McBride, M. B.; Environmental Chemistry of Soil; $1^{\text {st }}$ ed., Oxford University Press: New York, 1994.

15. Pierangeli, M. A. P.; Guilherme, L. R. G.; Curi, N.; Anderson, S. J.; Lima, J. M.; Rev. Bras. Ciênc. Solo 2004, 28, 377.

16. Empresa Brasileira de Pesquisa Agropecuária; Manual de métodos de análise de solo, $2^{\text {a }}$ ed., Centro Nacional de Pesquisa em Solos: Rio de Janeiro, 1997.

17. Vettori, L.; Métodos de análise de solos, $1^{\mathrm{a}} \mathrm{ed}$., Ministério da Agricultura - Equipe de Pedologia e Fertilidade do Solo: Rio de Janeiro, 1969.

18. United States Environmental Protection Agency; Test methods for evaluating solid waste, physical/chemical methods, Rep. SW-846, Rev. 5., Office of Solid Waste and Emergency Response: Washington, DC, 1998.

19. Jackson, M. L.; Soil chemical analysis - advanced course, $2^{\text {nd }}$ ed., Prentice-Hall: Madison, 1979.

20. Lindsay, W. L.; Chemical equilibria in soils, $1^{\text {st }}$ ed., Wiley-Interscience: New York, 1979.

21. Menzies, N. W.; Fulton, I. M.; Morrell, W. J.; J. Environ. Qual. 2004, 33, 1877.

22. Altundogan, H. S.; Altundogan, S.; Tümen, F.; Memnune, B.; Waste Manag. 2000, 20, 761.

23. Cornell, R. M.; Schwertmann, U.; The Iron Oxides, $3^{\text {rd }}$ ed., Weinheim-VHC: New York, 2003.

24. Snars, K.; Gilkes, R.; Hughes, J.; Aust. J. Soil Res. 2003, 41, 1229.

25. Enick, R. M.; Beckman, E. J.; Shi, C.; Xu, J.; Energy Fuels 2001, 15, 256.

26. Oliveira, L. C. A.; Gonçalves, M.; Oliveira, D. Q. L.; Guarieiro, A. L. N.; Pereira, M. C.; Quim. Nova 2007, 30, 925.

27. Dias, N. M. P.; Alleoni, L. R. F.; Casagrande, J. C.; Camargo, O. A.; Rev. Bras. Ciênc. Solo 2001, 25, 297.

28. Pierangeli, M. A. P.; Guilherme, L. R. G.; Curi, N.; Silva, M. L. N.; Lima, J. M.; Costa, E. T. S.; Rev. Bras. Ciênc. Solo 2005, 29, 523.

29. Pierangeli, M. A. P.; Guilherme, L. R. G.; Curi, N.; Silva, M. L. N.; Oliveira, L. R.; Lima, J. M.; Rev. Bras. Ciênc. Solo 2001, 25, 269.

30. Pierangeli, M. A. P.; Guilherme, L. R. G.; Curi, N.; Costa, E. T. S.; Lima, J. M.; Marques, J. J. G. S. M.; Figueiredo, L. F. P.; Rev. Bras. Ciênc. Solo 2007, 31, 819 .

31. Pierangeli, M. A. P.; Guilherme, L. R. G.; Oliveira, L. R.; Curi, N.; Silva, M. L. N.; Pesq. agropec. bras. 2001, 36, 1077.

32. Pierangeli, M. A. P.; Guilherme, L. R. G.; Oliveira, L. R.; Curi, N.; Silva, M. L. N.; Pesq. agropec. bras. 2003, 38, 737.

33. Lide, D. R.; Handbook of chemistry and physics, $80^{\text {th }}$ ed., CRC Press: Boca Raton, 2000. 